\title{
Gastrocutaneous syndrome
}

INSERM

\section{Source}

INSERM. (1999). Orphanet: an online rare disease and orphan drug data base.

Gastrocutaneous syndrome. ORPHA:2069

Gastrocutaneous syndrome is a rare, syndromic, hyperpigmentation of the skin characterized by multiple lentigines and café-au-lait spots associated with hiatal hernia and peptic ulcer, hypertelorism and myopia. There have been no further descriptions in the literature since 1982. 